\title{
EPISTEMOLOGY AND THE STRUCTURE OF LANGUAGE
}

\author{
JEFFREY A. BARRETT ${ }^{1}$ \\ TRAVIS LACROIX ${ }^{1,2}$
}

\begin{abstract}
We are concerned here with how structural properties of language may come to reflect features of the world in which it evolves. As a concrete example, we will consider how a simple term language might evolve to support the principle of indifference over state descriptions in that language. The point is not that one is justified in applying the principle of indifference to state descriptions in natural language. Instead, it is that one should expect a language that has evolved in the context of facilitating successful action to reflect probabilistic features of the world in which it evolved.
\end{abstract}

\section{INTRODUCTION}

A simple term language might evolve to allow for effective communication in the context of a Lewis-Skyrms signaling game (Lewis, 1969: Skyrms, 2006, 2010a). Here we are concerned with how such an evolved language may reflect probabilistic features of the world in which it evolved.

Specifically, we will consider how a simple evolutionary process might lead to a language where unbiased priors over state expressions in the language accurately represent the relative frequencies of the corresponding states of nature; this is a howpossibly explanation (Hempel, 1965, Resnik, 1991) DWe examine how the structure of a language might evolve to represent some statistical properties of the world in such a way that a purported principle of rationality - namely, the principle of indifference - actually obtains. The suggestion is not that there is evolutionary warrant for adopting a principle of indifference $\mathrm{I}^{2}$ The point, rather, is that one should

$\left({ }^{1}\right)$ Department of Logic and Philosophy of Science, University of California, Irvine

$\left({ }^{2}\right)$ Mila, (Québec AI Institute / Institut QuéBÉcois D’Intelligence Artificielle)

E-mail address: j.barrett@uci.edu , tlacroix@uci.edu (corresponding author).

Date: January 2020. This is a pre-print of an article published in Erkenntnis. The final version is available online at: doi.org/10.1007/s10670-020-00225-4

${ }^{1}$ There is a tradition of such explanations in the field. Skyrms (2010a) shows how communication may evolve without natural salience, but not that it actually did so; Barrett (2007) and Purves (2018) show that it is possible for partitioning into linguistic kinds to evolve and to be ideally successful without latching onto natural kinds, but not that linguistic kinds in fact fail to track natural kinds; and Roth and Erev (1995); Erev and Roth (1998); Barrett and Zollman (2009) show that dynamics like forgetting and experimenting may promote the evolution of language, but not that they actually have been causal factors in the evolution of language.

${ }^{2}$ Though, see the discussion in Huemer 2009); Bradley (2019); Neth 2019). 
expect a language that has evolved in the context of facilitating successful action to reflect statistical features of the world in which it evolved. In the present case, it does so in a way that supports a purported epistemic principle. That said, what these features are more generally may be both subtle and difficult to determine.

We will start by considering how a language might evolve in the context of a signaling game. We will then consider how such an evolved language might come to reflect probabilistic properties of the world in which it evolved.

\section{Basic Signaling and the Problem of Priors}

In a basic two-agent $2 \times 2 \times 2$ Lewis-Skyrms signaling game, there are two states of nature, two types of signal, and two types of action-one matching each state of nature. On a play of the game, one agent (the sender) sees the state of nature, then chooses and sends one of the signals to the other agent (the receiver) who then performs an action. The action is successful if and only if it matches the current state of nature.

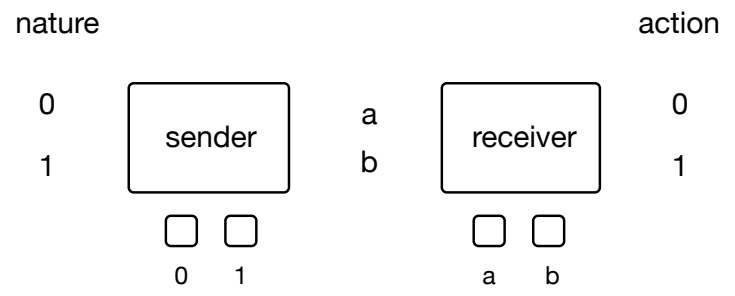

Figure 1. A basic $2 \times 2 \times 2$ signaling game

The two agents may evolve a signaling system over repeated plays of the game. One way that this might occur is under simple reinforcement learning. Suppose that the sender has two urns - one corresponding to each state of nature - and suppose that each urn initially contains one ball of each of the two signal types. Similarly, suppose that the receiver has two urns - one corresponding to each possible signaland suppose that each urn initially contains one ball of each of the two action types. When the sender sees the current state of nature, she goes to her corresponding urn, draws a ball at random, then sends the signal indicated by the ball. When the receiver sees the signal, he goes to his corresponding urn, draws a ball at random, and performs the action indicated by the ball. On simple reinforcement learning, if the action matches the current state of nature (and is hence successful), then the agents each return the ball they drew to the urn from which they drew it and add another ball of the same type to that urn, thus making their successful action more probable given the antecedent state or signal type. Otherwise, they just return the ball they drew to the urn from which they drew it. 
If nature is unbiased, then the basic $2 \times 2 \times 2$ signaling game will converge to an optimal signaling system with probability one under this simple dynamics ${ }^{3}$ Convergence to a signaling system still occurs, but is less likely, under simple reinforcement learning for an $n \times n \times n$ signaling game the larger $n>2$ and the more biased the distribution of states of nature.

Language users may learn something concerning the nature of the world they inhabit as they evolve a language appropriate for describing their world. Consider a sender-predictor game, a slight variant of the signaling game just described ${ }^{4}$ Suppose that the state of nature is observed in the morning and that the action taken by the receiver is a predictive action in the afternoon. One morning state might be clear skies, with the corresponding successful afternoon action being take straw hats to the picnic (because sun is more likely in the afternoon when it is clear in the morning). The other morning state might be cloudy skies, with the corresponding successful afternoon action being take umbrellas to the picnic (because rain is more likely in the afternoon when it is cloudy in the morning). Suppose, in the present case, that a morning state does not guarantee the corresponding afternoon state - it just makes it probable. Finally, suppose the sender tracks how often each of her terms, in fact, leads to successful action given the correlation between morning and afternoon states.

In this game, under simple reinforcement learning, if the morning state determines the probability of each afternoon state, then the sender will typically both evolve a successful signaling language and learn the probabilities of the receiver's prediction being true given the signal she sent him. Here precisely the same experience that allows her to evolve a successful descriptive language may also provide her with reliable estimates that might serve as effective priors for the receiver's predictions $5^{5}$

There is a sense in which one might take such a story to solve the problem of priors - the worry that a belief revision model of knowledge requires one to assign prior probabilities that are then updated over time, when there is no agreed-upon rational principle for how to initially assign prior probabilities. In the model just described, reliable, effective priors for success, given a description of the current state in the evolved language, co-evolve with the meanings of such descriptions. Indeed, if the agents evolving the language pay attention to how well it works as they evolve it, the 'prior' they assign to successful action, given a description of the current state, is not well understood as a prior at all. Rather, such 'priors' are expectations that are grounded in the long experience that led to the evolution

${ }^{3}$ See Argiento et al. (2009) for details.

${ }^{4}$ See Barrett (2014b) for a discussion of such games more generally.

${ }^{5}$ See Barrett (2014a) for a detailed description of this particular sender-predictor game, its behavior, a discussion of the problem of priors, and a proposed dissolution of the problem. 
of a useful predictive language. On this view, one never faces the problem of assigning prior probabilities to descriptions with no relevant background knowledge whatsoever. If one is ever assigning probabilities over evolved descriptions, then one already knows how to use a language that was forged in the context of success and failure in action, which means that one also has substantial knowledge regarding the world and how it works.

Here, in contrast, we will consider how the structure of an evolved language may come to mirror the structure of the world in which it evolved. Specifically, we will consider how a simple term language may evolve under plausible generic conditions to make unbiased priors over descriptions in the language reliable. Again, the point is not that one is in any way justified in accepting the principle of indifference over a natural language. Rather, it is that a language that evolves in the context of its usefulness will mirror features of the world where it evolved. Even so, while they may know well that their evolved language facilitates successful action, there is no reason to suppose that the agents who use it will know precisely how its structure mirrors their world 6

\section{The Principle of Indifference}

The principle of indifference says that, in the absence of other information, one should assign probability $1 / n$ to each of $n$ specifiable possibilities. If one's language individuates between six sides of a die, for example, the principle would recommend that one assign a probability of $1 / 6$ to each possible outcome on this representation. On its face, this principle tells one how to assign prior probabilities and so appears to provide a solution to the problem of priors; however, implementing a solution along these lines poses serious conceptual difficulties.

As a basic principle of reason, the principle of indifference has little to recommend it. As indicated by how we just characterized the principle, it depends on how one individuates between states. This, in turn, depends on one's language and associated conceptual scheme. The problem is that the principle of indifference typically provides different recommendations for how one ought to assign probabilities to a given possibility for different partitions of possibilities. Under the description that the outcome of a toss of the six-sided die might be any of six possibilities one to six, the principle would recommend assigning probability $1 / 6$ to getting a one. But under the description that the outcome of the toss might be the number one or not the number one, the principle would recommend assigning probability $1 / 2$ to getting the outcome the number one and probability $1 / 2$ to getting the outcome not the number one. If one takes there to be a matter of fact regarding whether a

${ }^{6}$ See Barrett 2007) and Purves 2018) for explanations of this sort of failure. 
probability assignment is reliable, then at most one of these recommendations can be right.

One might object that the second partition (between the number one or not the number one) is less natural than the first (between each of the six possibilities one to six). But the naturalness of a partition depends on one's background commitments, the context at hand, and the specific representational features of one's language. If one happened to believe that the die was weighted in such a way that was strongly biased toward the number one, then the second partition might well seem the more natural.

Bas van Fraassen's (1989) cube factory story illustrates the general point $7^{7}$ Suppose one only knows that a factory produces cubes with a side between 0 and 2 meters. If one considers side-length, one might imagine that a principle of indifference requires that one take the probability of a randomly selected cube having a side between 0 and 1 to be $1 / 2$, since side-lengths range from 0 to 2 . If one considers volume, one might imagine that a principle of indifference requires that one take the probability of a randomly selected cube having a volume between 0 and 1 to be $1 / 4$, since volumes range from 0 to 4 . But since having a side length between 0 and 1 is the same thing as having a volume between 0 and 1 , the different partitions, each perfectly natural given different interests, yield inconsistent probability assignments.

The moral here has two parts: (1) the principle of indifference makes no recommendations whatsoever without a specified partition since different partitions yield inconsistent probability assignments, and (2) the partition that one finds most natural will depend on one's interests and the representational structure of one's language.

If the principle of indifference were in fact a basic principle of reason, something should go wrong if an agent were not to use it. It is worth noting, however, that it is entirely unclear what such bad consequences might be. A good Bayesian may assign any set of coherent, non-dogmatic priors (that is, probabilities that satisfy the standard axioms of probability theory and are neither zero nor one) to her $n$ hypotheses without fear of finding herself committed to a Dutch Book or failing to respond appropriately to relevant evidence. And, as she conditions on evidence, she will expect that her degrees of belief will reflect relative frequencies in nature.8

That said, a language may evolve in such a way that it supports the principle of indifference. More specifically, the terms in the evolved language may come to partition nature in such a way that the principle of indifference assigns probabilities

${ }_{7 \text { See also Bertrand }}(1889)$.

${ }^{8}$ Another well-known problem with the principle of indifference is that it leads to complications and inconsistencies on infinite domains (Keynes 1921). This will not concern us here, given that all of our examples are finite. 
over the partitions that are, in fact, approximately equal to the observed relative frequency of each type of state in nature. Here we will consider one way in which this might happen. This illustrates how statistical facts concerning the nature of the world might condition the structure of a language that evolves by way of success and failure in the context of that world.

\section{The Evolution of Indifference}

Consider a signaling game just like the $2 \times 2 \times 2$ game above, but where there are more states of nature and corresponding acts than there are expressions that the sender might use to signal the receiver. Here the agents lack the expressive resources to evolve a language that perfectly communicates the current state of nature. While an evolved language will never be perfectly successful, it might be more or less optimal given the agents' expressive constraints.

Note that there are several extensions of the basic signaling game which we do not consider here. One might, for example, consider a version of the game where there are more terms than there are relevant states, giving rise to synonyms namely, states may come to be represented by more than one signal so that the sender strategy is one-to-many (Skyrms, 2010a; Hu et al., 2011). In games like this, terms are rarely equiprobable. Here players may evolve to fully represent nature with their communicative resources. This is not true when there are more states than signals.

The syntactic games considered in Barrett (2006, 2007, 2009) also provide models where the evolved language may partition nature. However, concatenating signals in such games, again, provides a means for fully representing the states of nature.

There are many ways in which language may evolve to partition nature. Not all of these will be relevant for a principle of indifference ${ }^{9}$ Here we are concerned with a simple model for how language may evolve to partition nature in cases where the agents do not have sufficient linguistic resources to fully represent the states of nature. As we will see, this model may lead to a language that partitions nature in a way that supports a principle of indifference.

When there are more states than terms, a language may evolve where each state of nature triggers the sender to send a particular corresponding term. If so, the evolved terms partition the states of nature. Each term is associated with an element of the partition containing the states that correspond to that term.

The most efficient signaling system the agents might evolve would be one that communicates the most information about the current state of nature per signal ${ }^{10}$

${ }^{9}$ See also O'Connor 2017), who discusses interactions between structures in the world and structures in language in signaling games, and Purves (2018).

${ }^{10}$ This is sometimes referred to as the maximum entropy principle. It is closely tied to the principle of indifference, but for reasons discussed in the last section and later in the paper, we do not take 
The mean self-information of a signal on a partition of nature is given by

$$
\sum_{i}-p_{i} \log p_{i}
$$

where $p_{i}$ is the probability associated with element $i$ of the partition. This expression is maximal when the probabilities corresponding to each element of the partition are equal. The agents' evolved language communicates the most information per signal if the probability of each type of signal is equal; this occurs when the signals partition the states of nature into equally probable sets 11

Since a language where the terms partition nature into equally probable sets allows the agents to communicate the most information per signal, one might expect such a language to evolve in a special context where agents are somehow rewarded for precisely this sort of efficient communication. But a special efficiency reward is not a necessary condition for the evolution of a simple, efficient term language. Indeed, languages that induce an unbiased partition over states often evolve in the context of simple reinforcement learning alone. And when the induced partition is not precisely unbiased, it is typically very close. That this occurs is perhaps particularly salient given the simplicity of reinforcement learning and the fact that it is ubiquitous in nature ${ }^{12}$

4.1. Unbiased States. We will start with an unbiased $10 \times 2 \times 10$ signaling game (a game with ten equiprobable states of nature, two terms, and ten acts, one corresponding to each of the states). Here the sender has ten urns, one for each state of nature, each starting with one ball of each of the two signal types, and the receiver has two urns, one for each signal type, each starting with one ball for each of the nine act types. As above, we will suppose that both the sender and receiver learn by simple reinforcement. Since each type of natural state is equiprobable, an evolved language on a $10 \times 2 \times 10$ signaling game will partition the states into equiprobable sets if and only if each term comes to be triggered by precisely five of the types of state. We will represent this partition as $(5,5)$ and the other possible partitions similarly.

The chance payoff for this game is $1 / 10$, and the maximal payoff, if the agents evolve a perfectly effective term language, is $1 / 5{ }^{13}$ Over 1000 runs with $10^{6}$ plays

maximum entropy to provide any justification whatsoever for adopting the principle of indifference. See Jaynes (1957); Williamson (2010) for contrary views on this issue.

${ }^{11}$ See Shannon (1948); Shannon and Weaver (1949). For a careful analysis of information in the context of signaling games, see Skyrms (2010a b).

${ }^{12}$ For empirical work on reinforcement learning in nature, see Rescorla and Wagner (1972); Roth and Erev (1995); Schultz et al. (1997); Erev and Roth (1998); Schultz (2004). For an introduction to reinforcement learning in a computational framework, see Sutton and Barto (1998).

${ }^{13}$ Every partition that takes advantage of both signals, that is, every partition except $(0,10)$ and $(10,0)$ — which are dynamically unstable - has the same expected payoff as long as the sender and 


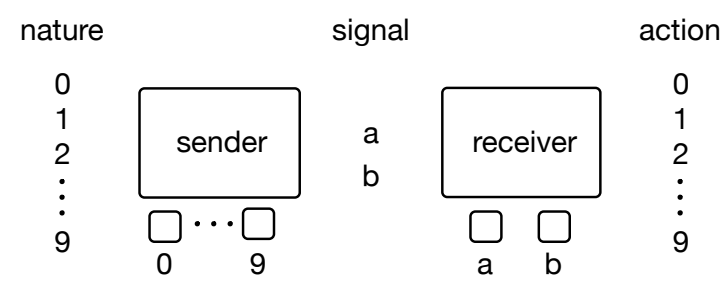

FIGURE 2. evolving an unbiased partition

per run, the average expected payoff at the end of each run is $0.1999{ }^{14}$ This convergence is relatively quick: after 4853 plays, on average, the agents are within $5 \%$ of their final expected payoff ${ }^{15}$ Most runs (0.875) achieve a cumulative success rate of at least 0.199016

The unbiased $(5,5)$ partition is indeed most common of the 9 stable partitions. Nearly $1 / 3$ of the time (0.310), the sender and receiver perfectly partition nature for maximal information transfer on the equiprobable $(5,5)$ partition. And most of the time (0.792), the sender and receiver partition nature near-perfectly by evolving a $(5,5),(6,4)$, or $(4,6)$ partition. While it is rare, the sender and receiver sometimes (0.031) fail to evolve a clear partition of nature on $10^{6}$ plays.

Figure 3 gives the distribution of runs for the $10 \times 2 \times 10$ game under simple reinforcement. The dashed line represents the mean information per signal over the distribution, and the smooth line represents the number of ways that each type of partition might be achieved. A natural explanation for why simple reinforcement typically leads to a term language that induces an equiprobable or nearly equiprobable partition over the possible states of nature is that there are simply more ways to get such a partition given a maximally successful language. That said, the likelihood of less even partitions drops off even faster than the number of ways to get the less even partitions. A Kolmogorov-Smirnov (K-S) test of the empirical distribution against a distribution sampled from the combinatorial expectation yields a $p$-value

receiver coordinate upon the partition and regardless of what weight the receiver puts on each action conditional upon the relevant signal. See LaCroix (2020) for details.

${ }^{14}$ The average expected payoff is calculated from the evolved dispositions of the agents (that is, from their actual urn contents) at the end of the run given the unbiased (or biased as in the next section) states of nature.

${ }^{15}$ The fastest run achieves this payoff within 1801 plays and the slowest after 25995 plays. 0.90 runs are within $5 \%$ of their final expected payoff prior to the first 7500 plays.

${ }^{16}$ The cumulative success rate is a measure of success that takes account of the history of the game. It is calculated by dividing the number of plays that led to a success by the total number of plays in that run. When the players are successful, early failures are washed out as the number of plays increases. 


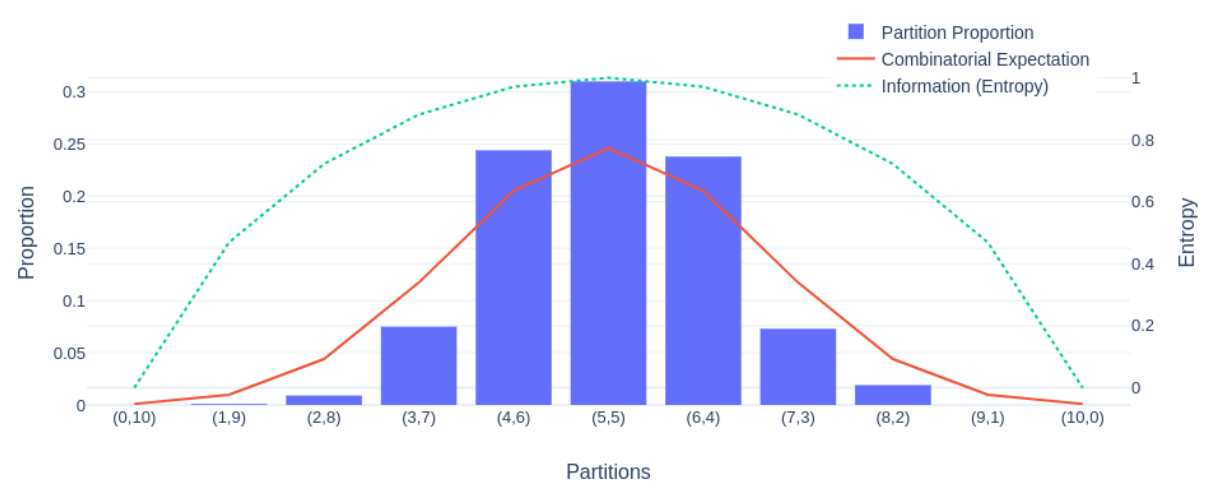

Figure 3. Partitioning 10 states with 2 signals with unbiased nature. Comparison of experimental results with combinatorial expectation and information transfer

of 0.0017 for the $10 \times 2 \times 10$ signaling game, suggesting that the combinatorial expectations do not by themselves explain the empirical results. See Figure $4{ }^{17}$

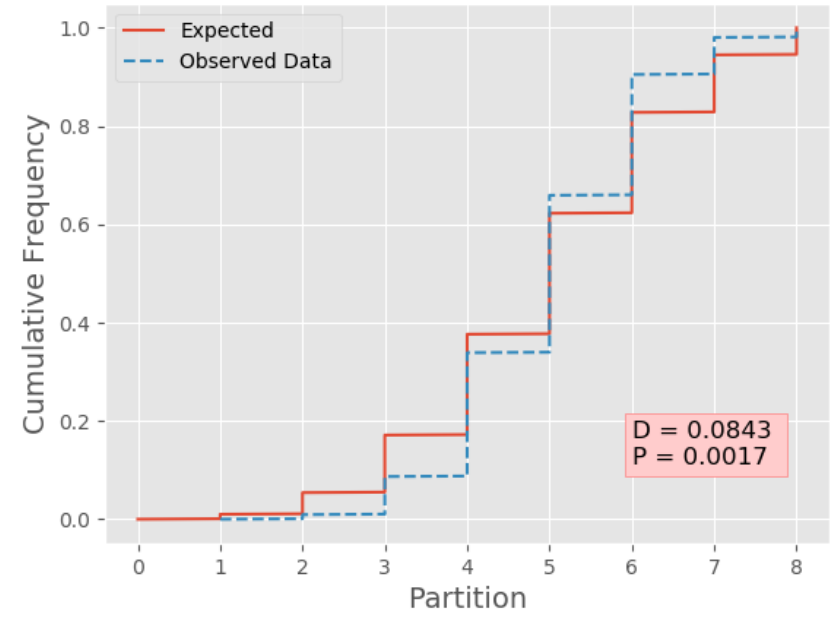

Figure 4. Comparison of the CDFs for our observed data and the theoretical expectation

\footnotetext{
${ }^{17}$ To provide a bit more detail, the null hypothesis is that the combinatorial distribution and the empirical distribution are identical for the $10 \times 2 \times 10$ signaling game. The K-S test gives the statistic $D=0.0843$, which is the supremum of the set of distances between the empirical distribution function we observe and the expected distribution function from the combinatorial measure. This corresponds to a $p$-value of 0.0017 ; hence one might reject the null hypothesis with high confidence.
} 
One observes the same phenomena in the unbiased $9 \times 3 \times 9$ signaling game (with nine equiprobable states of nature, three terms, and nine acts, one corresponding to each of the states). Suppose again that both the sender and receiver learn by simple reinforcement.

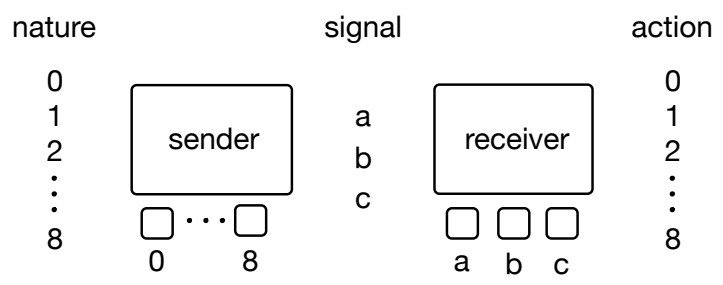

FiguRE 5. evolving an unbiased partition

Here, the chance payoff for the $9 \times 3 \times 9$ game is $1 / 9$, and the maximal expected payoff is $1 / 3$. On 1000 runs with $10^{6}$ plays per run, the average expected payoff at the end of a run is 0.3332 . The vast majority of runs (0.926) achieve a near-perfect expected payoff greater than 0.333, and every run (1.000) achieves an expected payoff greater than 0.330. Moreover, learning is fast: after 6495 plays, on average, the agents are within $5 \%$ of their final expected payoff ${ }^{18}$ The cumulative success rate is 0.3323 after $10^{6}$ plays per run, on average (1000 runs), and almost every (0.990) run achieved a cumulative success rate of at least 0.330 .

Again, the unbiased $(3,3,3)$ partition is most common of the 28 stable partitions. About $1 / 6$ of the time $(0.160)$, the sender and receiver perfectly partition nature for maximal information transfer. And more than $2 / 3$ of the time (0.684), the sender and receiver partition nature near-perfectly by evolving either the $(3,3,3)$ partition or one of the six permutations of the $(4,3,2)$ partition. Very rarely $(0.031)$, the sender and receiver fail to evolve a clear partition of nature.

Figure 5 illustrates the results of the simulation. Note again that the game tracks equiprobable partitions even better than the combinatorial measure over the number of ways of getting each type of partition. These results are more disparate than in the $10 \times 2 \times 10$ game ${ }^{19}$ In this spirit, the results in the next section further illustrate, in a dramatic way, why the combinatorial measure alone cannot be used to explain the partitions that evolve under simple reinforcement.

4.2. Biased States. The natural next step is to consider whether a term language might evolve under simple reinforcement to partition states into equiprobable sets

\footnotetext{
${ }^{18} 0.90$ runs are within $5 \%$ of their final expected payoff prior to the first 9500 plays.

${ }^{19} \mathrm{~A} \mathrm{~K}-\mathrm{S}$ test of the empirical distribution against a distribution sampled from the combinatorial expectation yields the statistic $D=0.1912$, with a $p$-value $p<0.0001$, suggesting again that the combinatorial expectations do not by themselves explain the empirical results.
} 


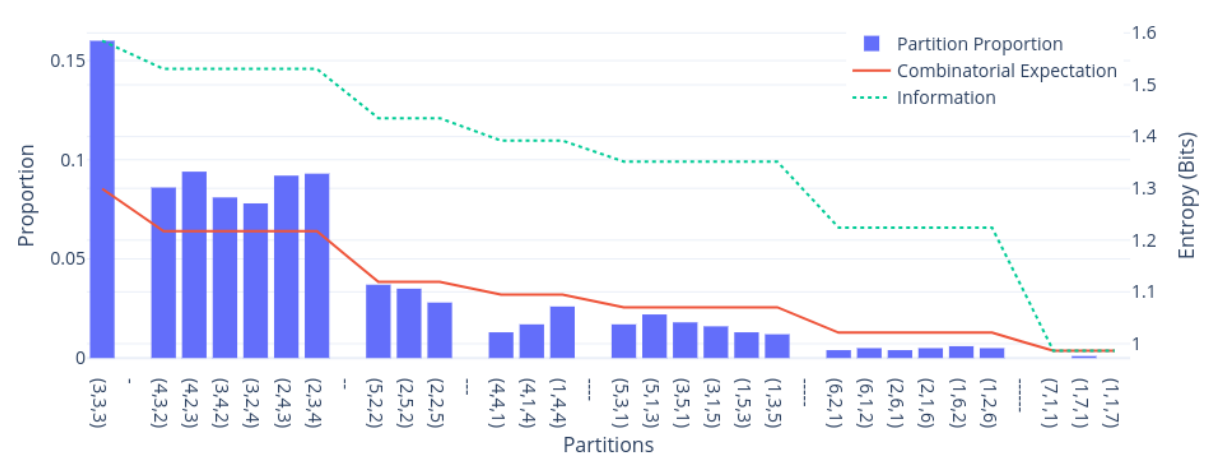

Figure 6. Partitioning 9 states with 3 signals with unbiased nature. Comparison of experimental results with combinatorial expectation and information transfer

when those states are not themselves equiprobable. This is what would be required for the principle of indifference to be applicable in any robust sense for the present model. Note that if this is true, then it cannot be the number of ways of achieving a particular partition that drives the evolution of partitions as the equiprobable partitions over strongly biased states are correspondingly special.

Consider the $10 \times 2 \times 10$ game where nature is biased as indicated by the vector

$$
\langle 1 / 2,1 / 18,1 / 18,1 / 18,1 / 18,1 / 18,1 / 18,1 / 18,1 / 18,1 / 18\rangle
$$

for states 0 through 9 respectively. If signals and actions are themselves random with no bias, then the expected chance payoff is 0.10 , and if the two signals are used optimally, then the maximal expected payoff is $10 / 18 \approx 0.5556$.

On simulation under simple reinforcement learning, the expected payoff given the evolved urn contents is, on average, 0.5502 after $10^{6}$ plays per run over 1000 runs. Many runs (0.8989) achieve an expected payoff of better than 0.55 with this number of plays. Convergence here is relatively fast. After 1999 plays, on average, the agents are within $5 \%$ of their final expected payoff ${ }^{20}$ The cumulative success rate is, on average, 0.5496 after $10^{6}$ plays, and many runs (0.887) achieve a cumulative success better than 0.55 .

Most of the time (0.897), the players learn to partition nature, and they almost always evolve an unbiased, or nearly unbiased, partition over the states. On the sender end, the most common partition (0.570) is one where she uses one term for the most common state and the other for the other eight states. Of course,

${ }^{20} 0.90$ runs are within $5 \%$ of their final expected payoff prior to the first 2200 plays. 
which term evolves to be used for what on a particular run is entirely random. Of these runs where the players do learn to partition nature, about $1 / 4$ of the time (0.281), she pools a second state into the signal containing the biased state. Sometimes (0.082), she pools two extra states into the signal containing the biased state. And rarely (0.008), she pools three extra states into the signal containing the biased state for a $(4,6)$ or $(6,4)$ partition, with approximately equal frequency. Given this particular natural bias, we never see the sender evolve a $(5,5)$ partition on simulation. On the receiver end, when the sender pools an extra state into the signal that contains the biased state, the receiver always chooses the action corresponding to the biased state when he sees that signal.

Given the strength of the bias and the simplicity of the learning dynamics, it is perhaps unsurprising that the receiver sometimes (0.083) learns to completely ignore the signal and just do the action corresponding to the biased state. Here the players cannot expect to do better than a success rate of 0.5. Sometimes (0.020), we see the receiver putting nearly full weight on the action corresponding to the biased state for one signal type and mixing over the biased state and other states on the other signal type.

One sees similar behavior in the biased $9 \times 3 \times 9$ game. Consider the natural bias

$$
\langle 1 / 3,1 / 3,1 / 21,1 / 21,1 / 21,1 / 21,1 / 21,1 / 21,1 / 21\rangle
$$

over the nine states 0 through 8 . Here, the expected chance payoff for unbiased random signaling is $1 / 9$, and the maximal expected payoff is $15 / 21 \approx 0.7142$. On simulation, we see an average expected payoff of 0.6970 after $10^{6}$ plays per run over 1000 runs. Most runs (0.794) achieve an expected payoff of at least 0.71 after $10^{6}$ plays. And again, the evolutionary speed is good. After 5406 plays, on average, the agents are within $5 \%$ of their final expected payoff ${ }^{21}$ Most runs (0.742) achieve a cumulative success greater than 0.71 over $10^{6}$ plays.

Most of the time $(0.772)$, the sender and receiver evolve a clear partition of the states. Again, the evolved partition tends to be close to unbiased and hence close to optimal. The most common partition (0.347) is one where the sender assigns one biased state to one signal, the other biased state to the other signal, and the remaining seven states to the third signal. Next most often (0.325), the sender pools an extra state with one of the signals that contains one of the biased states. More biased natural partitions are increasingly less common. The least common partition is one where the sender assigns three states to each of the three terms ${ }^{22}$ Again, when the sender pools other states into the signal containing one of the biased

${ }^{21} 0.90$ runs are within $5 \%$ of their final expected payoff prior to the first 10000 plays.

${ }^{22} \mathrm{~A}(5,3,1)$ partition occurs 0.073 , a $(4,4,1)$ partition occurs 0.019 , a $(5,2,2)$ partition occurs 0.066 , a $(4,3,2)$ partition occurs 0.030 , and a $(3,3,3)$ partition occurs 0.005 . In each case, the distinct permutations of these partitions occur with roughly equal frequency. 
states, the receiver learns to ignore the action associated with these extra states and puts all weight for that signal onto the act associated with the biased state.

The agents sometimes fail to evolve a clear partition on $10^{6}$ plays. Sometimes (0.034), for example, the sender pools both of the biased actions into one signal, and the receiver mixes over the appropriate actions, given that signal. The receiver perfectly partitions the remaining seven states into the other two signals, and the receiver mixes over the appropriate actions, conditional upon the signal received. This strategy has an expected payoff of $9 / 21 \approx 0.4286$. Mixtures between this and the clear partition cases are also observed with somewhat higher expected success rates.

\section{Discussion}

These models illustrate how expressions in a simple term language might evolve to represent equiprobable partitions of the fine-grained states of nature in a simple evolutionary context. From the perspective of the evolved language, it will look like every natural possibility is equiprobable. While this is manifestly not the case for the fine-grained states in the biased models, it is nevertheless often the case for those models that every expressible possibility in the evolved language is equiprobable or nearly so. Recall from the discussion in Sections 2 and 3 that the principle of indifference, at first glance, provides a solution to the problem of priors insofar as it tells one how to assign prior probabilities. However, in our models, the principle of indifference holds over the partition induced by the evolved expressions not because each fine-grained state of nature is, in fact, equiprobable but because, under the special circumstances that obtain here, the language evolves to partition nature into expressible states that are equiprobable.

Of course, this does not mean that one should expect the principle of indifference to hold for natural language generally. Even the relatively simple signaling languages of animals are often much more complex and expressive than the sort of term languages we have been considering here ${ }^{23}$ With a more expressive language, one might characterize different partitions over natural states, and one may have every reason to suppose that the principle of indifference will give the wrong probabilities

\footnotetext{
${ }^{23}$ For example, the data presented in the original studies of Seyfarth et al. (1980a b) on vervet monkey alarm-call systems indicated substantial variation in responses and that the responses are probabilistic rather than deterministic. Furthermore, vervets do not just vocalize for alarm calls. They also call when they find food, in aggressive confrontations, and during sexual activity, among others. Vervets additionally vocalize via grunting in a variety of circumstances: (a) when a submissive meets a dominant individual, (b) when a dominant meets a submissive individual, (c) when one vervet goes out into an open area, and (d) when a vervet comes across an outgroup conspecific (Cheney and Seyfarth 1982, 1990). Schlenker et al. (2016) provide a linguistic analysis of approximately 40 years of data from experimental primatology, which displays several complexities of monkey communication systems. This highlights but some of the myriad ways in which the world is significantly more complex than the model which we use to represent it.
} 
for most of these. Even if one restricts consideration to simple term languages like those discussed here, the language may evolve by means of something other than simple reinforcement, and the resulting language may well not support the principle of indifference ${ }^{24}$ And even with simple reinforcement, as we have seen, the principle of indifference does not always work for the evolved language - it just works on most runs of the present special models.

The moral, then, is not that there is any evolutionary warrant for adopting the principle of indifference or for supposing that natural language usually evolves to be maximally informative. There isn't, and it doesn't. Rather, it is that however a natural language evolves, inasmuch as it is forged in the context of success and failure in action, those features of the world that helped to shape the language may be reflected in the evolved language's structure. Further, this may occur in such a way that the principle of indifference over the evolved linguistic partition itself evolves to be a reliable epistemic guide. As a language evolves, one should expect it come to reflect various bits of structure in the world in which it evolved. A language may be influenced by social factors (Lupyan and Dale, 2010) or environmental factors (Kemp et al. 2019) ${ }^{25}$ What we have shown are the conditions under which the structure of a language might evolve to represent statistical properties of the world in such a way that a purported principle of rationality in fact obtains. Put another way, we have shown how a purported principle of rationality may be reliable contingent upon the way the world is actually partitioned by the language used to describe that world. Concretely, we have shown the conditions under which the principle of indifference obtains.

If one knew how the structure of a language was influenced by the world it evolved in, one might infer properties of the world, like the relative frequencies of natural states in the present models, from the structure of the language. But, for real languages, these relations will be extremely subtle, as subtle as the evolutionary contexts where the language was forged. And one should not expect these relations to provide general principles of reason nor even any epistemic short cuts. Determining precisely how the contingent structure of a natural language may reflect the structure of the world in which it evolved is highly nontrivial. Here we have provided a concrete example of how one might study a very simple epistemic relationship between the structure of the world and the evolved structure of a basic signaling language.

${ }^{24}$ See Barrett and Zollman (2009) for several learning dynamics that typically behave very differently than simple reinforcement learning. As a quick and very simple example, the learning dynamics win-stay/lose-shift would not even evolve a stable language in the present games, let alone one that supported the principle of indifference.

${ }^{25}$ There is an extensive literature in cognitive science that seeks to quantify how language might reflect the structure of the world. See Lupyan and Dale (2016) for an overview and discussion of the linguistic niche hypothesis. 


\section{REFERENCES}

Argiento, R., Pemantle, R., Skyrms, B., and Volkov, S. (2009). Learning to Signal: Analysis of a Micro-Level Reinforcement Model. Stochastic Processes and Their Applications, 119:373-390.

Barrett, J. (2006). Numerical Simulations of the Lewis Signaling Game: Learning Strategies, Pooling Equilibria, and Evolution of Grammar. Technical Report. UC Irvine, Institute for Mathematical Behavioral Science. Preprint MBS 06-09.

Barrett, J. (2007). Dynamic Partitioning and the Conventionality of Kinds. Philosophy of Science, 74:527-546.

Barrett, J. (2009). Faithful Description and the Incommensurability of Evolved Languages. Philosophical Studies, 147(1):123-137.

Barrett, J. (2014a). Description and the Problem of Priors. Erkenntnis, 79(6):13431353.

Barrett, J. (2014b). On the Coevolution of Theory and Language and the Nature of Successful Inquiry. Erkenntnis, 79(Suppl 4):821-834.

Barrett, J. and Zollman, K. (2009). The Role of Forgetting in the Evolution and Learning of Language. Journal of Experimental and Theoretical Artificial Intelligence, 21(4):293-309.

Bertrand, J. (1889). Calcul des probabilités. Gauthier-Villars et Fils, Paris.

Bradley, D. (2019). Naturalness as a Constraint on Priors. Mind, Forthcoming.

Cheney, D. and Seyfarth, R. (1990). How monkeys See the World: Inside the Mind of Another Species. University of Chicago Press, Chicago.

Cheney, D. L. and Seyfarth, R. M. (1982). How Vervet Monkeys Perceive Their Grunts: Field Playback Experiments. Animal Behaviour, 30(3):739-751.

Erev, I. and Roth, A. E. (1998). Predicting How People Play Games: Reinforcement Learning in Experimental Games with Unique, Mixed Strategy Equilibria. The American Economic Review, 88(4):848-881.

Hempel, C. G. (1965). Aspects of Scientific Explanation, and Other Essays in the Philosophy of Science. Free Press, New York.

Hu, Y., Skyrms, B., and Tarrès, P. (2011). Reinforcement Learning in Signaling Game. arXiv preprint arXiv:1103.5818.

Huemer, M. (2009). Explanationist Aid for the Theory of Inductive Logic. British Journal for the Philosophy of Science, 60(2):345-375.

Jaynes, E. T. (1957). Information Theory and Statistical Mechanics. The Physical Review, 106(4):620-630.

Kemp, C., Gaby, A., and Regier, T. (2019). Season Naming and the Local Environment. In CogSci: The Annual Meeting of the Cognitive Science Society, pages $539-545$.

Keynes, J. M. (1921). A Treatise on Probability. Macmillan, London.

LaCroix, T. (2020). Communicative Bottlenecks Lead to Maximal Information Transfer. Journal of Experimental and Theoretical Artificial Intelligence. Forthcoming.

Lewis, D. (2002/1969). Convention: A Philosophical Study. Blackwell, Oxford.

Lupyan, G. and Dale, R. (2010). Language Structure is Partly Determined by Social Structure. PloS one, 5(1):e8559.

Lupyan, G. and Dale, R. (2016). Why Are There Different Languages? The Role of Adaptation in Linguistic Diversity. Trends in Cognitive Sciences, 20(9):649-660. 
Neth, S. (2019). Bayesian Naturalness. Unpublished Manuscript. April, 2019. PDF File.

O'Connor, C. (2017). Games and Kinds. The British Journal for the Philosophy of Science, 70(3):719-745.

Purves, G. M. (2018). Conventionality and Causality in Lewis-Type Evolutionary Prediction Games. The British Journal for the Philosophy of Science, axy076. Forthcoming.

Rescorla, R. A. and Wagner, A. R. (1972). A Theory of Pavlovian Conditions: Variations in the Effectiveness of Reinforcement and Nonreinforcement. In Black, A. H. and Prokasy, W. F., editors, Classical Conditioning II: Current Research and Theory, pages 64-99. Appleton-Century-Crofts, New York.

Resnik, D. B. (1991). How-Possibly Explanations in Biology. Acta Biotheoretica, 39(2):141-149.

Roth, A. and Erev, I. (1995). Learning in Extensive Form Games: Experimental Data and Simple Dynamical Models in the Intermediate Term. Games and Economic Behavior, 8:164-212.

Schlenker, P., Chemla, E., Schel, A. M., Fuller, J., Gautier, J.-P., Kuhn, J., Veselinović, D., Arnold, K., Cäsar, C., Keenan, S., Lemasson, A., Ouattara, K., Ryder, R., and Zuberbühler, K. (2016). Formal Monkey Linguistics. Theoretical Linguistics, 42(1-2):1-90.

Schultz, W. (2004). Neural Coding of Basic Reward Terms of Animal Learning Theory, Game Theory, Micro-economics and Behavioural Ecology. Current Opinion in Neurobiology, 14(2):139-147.

Schultz, W., Dayan, P., and Montague, P. R. (1997). A Neural Substrate of Prediction and Reward. Science, 275:1593-1599.

Seyfarth, R. M., Cheney, D. L., and Marler, P. (1980a). Monkey Responses to Three Different Alarm Calls: Evidence of Predator Classification and Semantic Communication. Science, 210:801-803.

Seyfarth, R. M., Cheney, D. L., and Marler, P. (1980b). Vervet Monkey Alarm Calls: Semantic Communication in a Free-Ranging Primate. Animal Behaviour, 28(4):1070-1094.

Shannon, C. (1948). A Mathematical Theory of Communication. The Bell System Mathematical Journal, 27:379-423.

Shannon, C. and Weaver, W. (1949). The Mathematical Theory of Communication. University of Illinois Press, Urbana and Chicago.

Skyrms, B. (2006). Signals. Philosophy of Science, 75(5):489-500.

Skyrms, B. (2010a). Signals: Evolution, Learning, $\&$ Information. Oxford University Press, Oxford.

Skyrms, B. (2010b). The Flow of Information in Signaling Games. Philosophical Studies, 147(1):155-165.

Sutton, R. S. and Barto, A. G. (1998). Reinforcement Learning: An Introduction. MIT Press, Cambridge.

van Fraassen, B. (1989). Laws and Symmetry. Clarendon Press, Oxford.

Williamson, J. (2010). In Defence of Objective Bayesianism. Oxford University Press, Oxford. 